\title{
HYBRIDIZATION OF SURFACE AND IMPURITY SPIN WAVES INDUCED BY THEIR PROPAGATION IN THE PLANE OF A THIN FERROMAGNETIC FILM
}

\author{
B. Kolodziejczak and H. PuszKarski \\ Surface Physics Division, Institute of Physics, A. Mickiewicz University \\ Matejki 48/49, Poznań 60-769, Poland \\ (Received September 29, 1991)
}

\begin{abstract}
Spin waves of a thin ferromagnetic film containing in its bulk two impurity layers, each parallel to the film surface and symmetrically disposed with regard to the film centre, are theoretically investigated within the framework of the Heisenberg model. The existence conditions for surface-localized and impurity-layer localized spin waves are established. Particular attention is given to the effect of a possible hybridization of these localized excitations; it is shown that this phenomenon does in fact occur for certain specified directions of the in-plane wave vector $k_{\|}$.
\end{abstract}

PACS numbers: $75.30 . \mathrm{Ds}, 75.70 .-\mathrm{i}$

\section{Introduction}

Recent interest in the theoretical study of magnetic systems with impurities arises from the concurrent development of new experimental and theoretical techniques for the study of these highly interesting systems. The use of ultra-high vacuum techniques permits the preparation of epitaxial magnetic films containing impurity layers sandwiched inside a single crystal. The resulting sandwiched film may present novel physical properties since the magnetic collective excitations occurring in a sandwiched film differ from those of its individual magnetic components in isolation. The most characteristic feature of the respective new magnetic spectrum consists in the existence of magnetic excitations (spin waves) localized on the impurity layer. It is the aim of the present work to study the existence conditions for such impurity-layer localized spin waves emerging in a magnetic thin 
film containing two impurity layers symmetrically disposed with respect to the centre (mid-plane) of the film. Our special interest will bear in particular on the coexistence conditions for surface-localized and impurity-layer localized spin waves and the investigation of their possible hybridization under certain conditions.

This paper may be regarded as an extension of our former papers $[1,2]$, where we studied a similar system; however, in the paper [2] we restricted our discussion from the very beginning to standing spin waves only. Here, the problem will concern spin waves with oblique wave vectors, i.e. ones which propagate in the plane of the film.

\section{The model}

In this paper we modify the model of the film considered in Ref. [1] by introducing into it two lattice planes with physical characteristics differing from those of the film; the planes will be referred to as "impurity planes". We make the following assumptions:

1. the impurity planes are disposed symmetrically with respect to the central plane of the film. Thus e.g. if the film consists of $L$ planes, labelled by $l$ from 0 to $L-1$, the impurity planes can occupy positions $N$ and $L-1-N$;

2. the boundary conditions (the surface anisotropy field) on the two surfaces of the film are assumed to be identical; interaction occurs between "nearest neighbouring" spins only;

3. the introduction of the impurity planes does not affect the crystallographical structure or the exchange integrals;

4. the sole difference in the physical conditions of a "host" spin and an impurity spin resides in the existence of a field $\boldsymbol{K}_{\mathbf{i}}$ acting on the impurities only (to be referred to as the "impurity anisotropy" field).

Thus, the Zeeman term of the Hamiltonian:

$$
\sum_{\ell j} \boldsymbol{H}_{\ell}^{\mathrm{eff}} \cdot \widehat{\boldsymbol{S}}_{\ell j}
$$

will involve an effective field $H_{\ell}^{\text {eff }}$ which we write in the form:

$$
\boldsymbol{H}_{\ell}^{\mathrm{eff}}=\boldsymbol{H}^{\mathrm{eff}}+\left(\delta_{0, \ell}+\delta_{\ell, L-1}\right) \boldsymbol{K}_{\mathrm{s}}+\left(\delta_{N, \ell}+\delta_{\ell, L-1-N}\right) \boldsymbol{K}_{\mathrm{i}} ;
$$

the exchange Heisenberg Hamiltonian is taken in the usual standard form. Some supplementary though hardly so important assumptions will be made in the course of our further considerations.

Our model may appear to be a highly simplified one. It is found, nonetheless, that even such "stripped" models can successfully describe (be it but qualitatively) real systems. This is all more important as recent progress in high vacuum technique permits the production of thin magnetic films with single impurity layer 
introduced into its bulk. Hence the necessity of theoretical studies of such simplified models aimed at elucidating the properties of the respective physical systems.

\section{The characteristic equation}

Following Ref. [1] we perform a sequence of spin transformations on the initial Hamiltonian thus obtaining a set of difference equations analogous to the sets (3.13-3.14) of Ref. [1]:

$$
\begin{aligned}
& {\left[R\left(k_{\|}\right)-E\left(k_{\|}, \tau\right)-\alpha\right] u_{0}(\tau)-2 S J_{1} \Gamma_{1} u_{1}(\tau)=0, \quad \ell=0,} \\
& -2 S J_{1}\left(\Gamma_{1}\right)^{*} u_{\ell-1}(\tau)+\left[R\left(k_{\|}\right)-E\left(k_{\|}, \tau\right)\right] u_{\ell}(\tau) \\
& -2 S J_{1} \Gamma_{1} u_{\ell+1}(\tau)=0, \quad \ell=1, \ldots, L-2, \\
& -2 S J_{1}\left(\Gamma_{1}\right)^{*} u_{L-2}(\tau)+\left[R\left(k_{\|}\right)-E\left(k_{\|}, \tau\right)-\alpha\right] u_{L-1}(\tau)=0, \quad \ell=L-1 .
\end{aligned}
$$

However, the assumptions underlying our model require that two equations of the set, namely those with $\ell=N$ and $\ell=L-1-N$, shall take a different form:

$$
\begin{aligned}
& -2 S J_{1}\left(\Gamma_{1}\right)^{*} u_{N-1}(\tau)+\left[R\left(k_{\|}\right)-E\left(k_{\|}, \tau\right)-\gamma\right] u_{N}(\tau) \\
& -2 S J_{1} \Gamma_{1} u_{N+1}(\tau)=0, \quad \ell=N, \\
& -2 S J_{1}\left(\Gamma_{1}\right)^{*} u_{L-2-N}(\tau)+\left[R\left(k_{\|}\right)-E\left(k_{\|}, \tau\right)-\gamma\right] u_{L-1-N}(\tau) \\
& -2 S J_{1} \Gamma_{1} u_{L-N}(\tau)=0, \quad \ell=L-1-N .
\end{aligned}
$$

The form of Eqs. (3) originates from our assumption that "closest neighbours" lie (at the farthest) in neighbouring planes. In the above equations $k_{\|} \equiv\left[k_{1}, k_{2}\right]$ denotes the in-plane wave vector, while $\tau \equiv k$ is a wave vector component perpendicular to the film surface; $u_{\ell}(\tau)$ are standing spin-wave mode amplitudes. The quantity $E\left(k_{\|}, \tau\right)$ is the energy of a given spin wave, and $\alpha$ and $\gamma$ are quantities related (respectively) to the surface and interface anisotropy fields. The quantity $\Gamma_{1}$ is a structural form factor (defined in Ref. [1]) depending on the in-plane wave vector $k_{\|}$. After some obvious transformations we arrive at the following set of equations identical with the set (3) of Ref. [2]:

$$
\begin{aligned}
& (x-a) u_{0}(k)-u_{1}(k) \\
& -u_{0}(k)+x u_{1}(k)-u_{2}(k) \\
& =0, \quad \ell=0 \text {, } \\
& =0, \quad \ell=1 \text {, } \\
& -u_{N-1}(k)+(x-c) u_{N}(k)-u_{N+1}(k) \\
& =0, \quad \ell=N \text {, } \\
& -u_{\ell-1}(k)+x u_{\ell}(k)-u_{\ell+1}(k) \\
& =0, \quad \ell=N+1, \ldots, \\
& L-2-N \text {, } \\
& -u_{L-2-N}(k)+(x-c) u_{L-1-N}(k)-u_{L-N}(k)=0, \quad \ell=L-1-N, \\
& -u_{L-3}(k)+x u_{L-2}(k)-u_{L-1}(k) \\
& -u_{L-2}(k)+(x-a) u_{L-1}(k) \\
& =0, \quad \ell=L-2, \\
& =0, \quad \ell=L-1 \text {, }
\end{aligned}
$$


where the energy (to within a multiplicative constant) is given by the expression:

$$
E\left(k_{\|}, k\right)=x-2\left|\Gamma_{1}\right| \cos (k),
$$

where $x$ is also a function of the in-plane wave vector $k_{\|}$. The explicit form of the relation is irrelevant for our further considerations. However, that of the $\boldsymbol{k}_{\|}$-dependence of the parameters $a$ and $c$ occurring in Eq. (4) is relevant. After trivial calculations, we obtain

$$
a=\frac{A_{1} z}{\left|\Gamma_{1}\right|}, \quad c=\frac{A_{3} z}{\left|\Gamma_{1}\right|},
$$

where $A_{1}$ and $A_{3}$ are phenomenological constants not dependent on $k_{\|}$and $k$, whereas $z$ denotes the number of "nearest neighbours" in the neighbouring plane.

It should be noted that with regard to our assumption of symmetric boundary conditions on the film the solutions can only be symmetric or antisymmetric. Having recourse to Ref. [2], we write the respective functions as follows: symmetric functions:

$$
\begin{array}{ll}
u_{\ell}(k)=\frac{\sin (\ell+1) k-a \sin (\ell k)}{\sin (N+1) k-a \sin (N k)}, & \ell=0, \ldots, N-1, \\
u_{\ell}(k)=\frac{\cos \left(\frac{L-1}{2}-\ell\right) k}{\cos \left(\frac{L-1}{2}-N\right) k}, & \ell=N, \ldots, L-1-N, \\
u_{\ell}(k)=\frac{\sin (L-\ell) k-a \sin (L-1-\ell) k}{\sin (N+1) k-a \sin (N k)}, & \ell=L-N, \ldots, L-1
\end{array}
$$

antisymmetric functions:

$$
\begin{array}{ll}
u_{\ell}(k)=\frac{\sin (\ell+1) k-a \sin (\ell k)}{\sin (N+1) k-a \sin (N k)}, & \ell=0, \ldots, N-1, \\
u_{\ell}(k)=\frac{\sin \left(\frac{L-1}{2}-\ell\right) k}{\sin \left(\frac{L-1}{2}-N\right) k}, & \ell=N, \ldots, L-1-N, \\
u_{\ell}(k)=-\frac{\sin (L-\ell) k-a \sin (L-1-\ell) k}{\sin (N+1) k-a \sin (N k)}, & \ell=L-N, \ldots, L-1 .
\end{array}
$$

The preceding expressions have still to be multiplied by a normalizing factor.

Only certain values of the wave-vector component $k$ perpendicular to the film surface are permitted; they are to be obtained from the following characteristic equations:

$$
2 \cos (k)-c-\frac{\sin (N k)-a \sin (N-1) k}{\sin (N+1) k-a \sin (N k)}=\frac{\cos \left(\frac{L-3}{2}-N\right) k}{\cos \left(\frac{L-1}{2}-N\right) k}
$$

in the case of symmetric solutions, whereas from

$$
2 \cos (k)-c-\frac{\sin (N k)-a \sin (N-1) k}{\sin (N+1) k-a \sin (N k)}=\frac{\sin \left(\frac{L-3}{2}-N\right) k}{\sin \left(\frac{L-1}{2}-N\right) k}
$$

in that of antisymmetric solutions.

Using (5) the energy $E\left(k_{\|}, k\right)$ is a real number only if $k$ assumes one of the following permitted forms:

$$
\begin{array}{ll}
k \in\langle 0, \pi\rangle, & \\
k=\mathrm{i} t, & t \in\langle 0,+\infty\rangle, \\
k=\pi+\mathrm{i} t, & t \in\langle 0,+\infty\rangle .
\end{array}
$$


Our primary interest will henceforth bear on the complex roots of the set (8). The solutions $u_{\ell}(k)$ of (4) corresponding to them are referred to as localized states. Depending on the relationship between the parameters $a, c$ and $N$, these states are localized on the impurities or surfaces. Since, here, $a$ and $c$ are parameterized by way of the vector $k_{\|}$, the last quantity will also intervene in the arisal and vanishing of localized states in our model. We shall concentrate on the changes taking place in the structure of energy spectrum and in the nature of states as dependent on variations of the vector $k_{\|}$; the variations in $k_{\|}$will be restricted to a fixed "path" lying within the 1st Brillouin zone.

\section{Structural functions}

We shall be studying the behaviour of the energy spectrum in conformity with the scheme proposed in Ref. [2], i.e. making use of the $c-a$ diagram (Fig. 1) permitting the immediate determination of the localization type of the states as well as their symmetry for a given combination of the parameters $a, c, N$ and $L$.

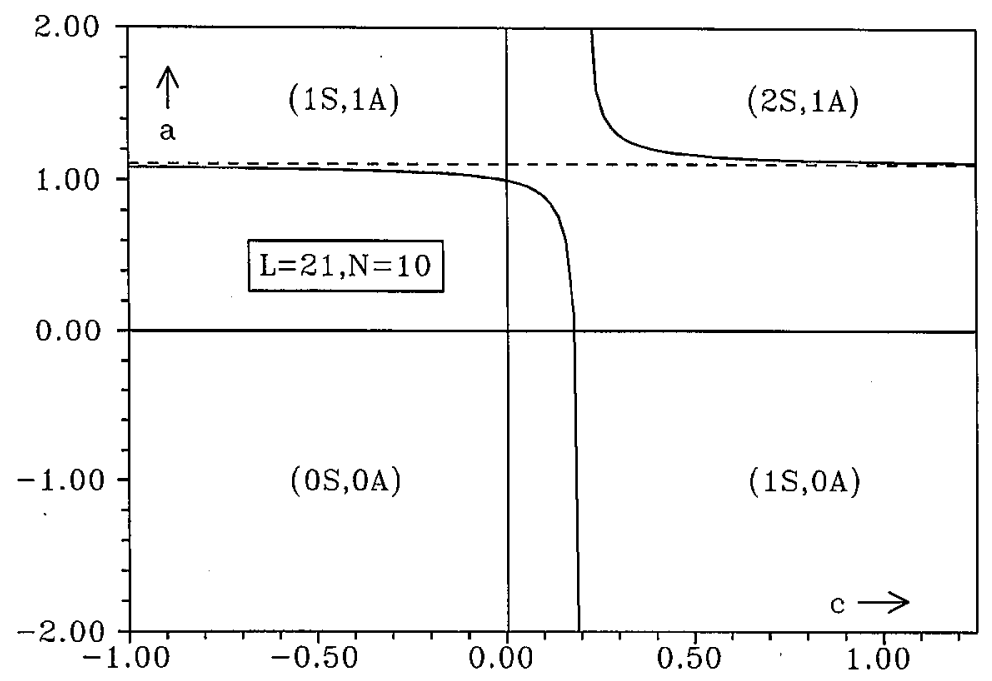

Fig. 1. The regions of existence of localized states in the $c-a$ plane. The hyperbola shown here is given by the equation $[a-(L+1) /(L-1)][c-2 /(L-1)]=[2 /(L-1)]^{2}$. The symbol $(n \mathrm{~S}, m \mathrm{~A})$ denotes that, in a given region, $n$ localized symmetric and $m$ localized antisymmetric states occur. Values assumed: $L=21, N=10$.

The form of the functions $a\left(k_{\|}\right)$and $c\left(k_{\|}\right)$(see Eq. (6)) is such that while varying $\left|\Gamma_{1}\right|$ continuously from $z$ to 0 one obtains a semi-straight line in the $c-a$ plane (referred to as the "trajectory") originating in the point $\left(A_{3}, A_{1}\right)$ (assuming that $\left.A_{1} A_{3} \neq 0\right)$ and lying on the straight line traversing the origin of coordinates with steepness given by the ratio $A_{1} / A_{3}$ and exhibiting the property of being contained completely within the quadrant to which the point $\left(A_{3}, A_{1}\right)$ belongs in 
which the semi-straight line begins. The considerations of Ref. [3] reveal certain essential properties of the function $\left|\Gamma_{1}\right|$. First, it is continuous; hence, on a closed region such as the Brillouin zone it is bounded and attains its limits. Its maximal value is just $z$ and its minimal value is 0 . We note that for known values of $A_{1}$, $A_{3}, N$ and $L$ Eqs. (8) for $a\left(k_{\|}\right)$and $c\left(k_{\|}\right)$permit the calculation of those values of $\left|\Gamma_{1}\right|$ for which the trajectory intersects the hyperbolae determining the boundaries of the regions of existence of localized states, i.e. we are in a position to determine exactly those vectors $k_{\|}$on the zone for which localized states will emerge. Hence it results that the boundaries between the regions of occurrence of localized states in the Brillouin zone represent respective "isohypses" of the function $\left|\Gamma_{1}\right|$.

It is noteworthy that the explicit form of the function $\left|\Gamma_{1}\right|$ is less essential, affecting only the dynamics of the arisal of localized states in its dependence on the vector $k_{\|}$but not affecting the structure of the energy spectrum as a whole when varying $\left|\Gamma_{1}\right|$ from its maximal value $\mathrm{z}$ down to its minimal value 0 . For this reason, we shall refrain from studying different functions $\left|\Gamma_{1}\right|$ corresponding to different crystallographical structures, but shall rather concentrate on one structure for which we expect to obtain results of some interest. We have in mind a thin film cut out of a simple cubic lattice with $\{111\}$ surface. This qhoice, in addition to the highly interesting form of $\left|\Gamma_{1}\right|$ presents the advantage that the term $x$ of Eq. (5) is not dependent on $k_{\|}$and the spectrum is not modulated by a component translating its structure homogeneously as a whole.

The function $\left|\Gamma_{1}\right|$ is now given as:

$$
\left|\Gamma_{1}\right|=\sqrt{3+2\left[\cos \left(k_{1}\right)+\cos \left(k_{2}\right)+\cos \left(k_{1}+k_{2}\right)\right]} .
$$

The surface, corresponding to the function (9), spanned over the Brillouin zone, is shown in Fig. 2. Since we shall be studying changes in the structure of the energy spectrum with varying $k_{\|}$, we visualize in Fig. 3 the fixed "path" in the zone along which $k_{\|}$varies, whereas Fig. 4 shows the changes in $\left|\Gamma_{1}\right|$ for $k_{\|}$varying as in Fig. 3 (conveying quantitative information - not only qualitative, as it was in the case of Fig. 2).

\section{Hybridization of the localized states}

One of the most interesting properties of the model with impurities resides in the fact that it admits of the phenomenon of hybridization between localized states. We shall refrain from discussing the conditions and consequences of the phenomenon referring for the details to Refs. [4] and [5]. Hybridization of two energy levels is recognizable by their coming sufficiently close to each other for the mutual perturbation in their energies to become significant (this requires a change at least in one of the physical factors involved). According to Ref. [5] hybridization is apparent on the energy graph as "repulsion" between the respective energy levels, with the requirement that the states corresponding to these levels shall possess the same symmetry. Hence the conclusion that hybridization leads to an "exchange" in the type of localization of the states involved; if a given state was originally localized, say, on a surface, after hybridization it becomes localized on impurity planes, and vice versa. 


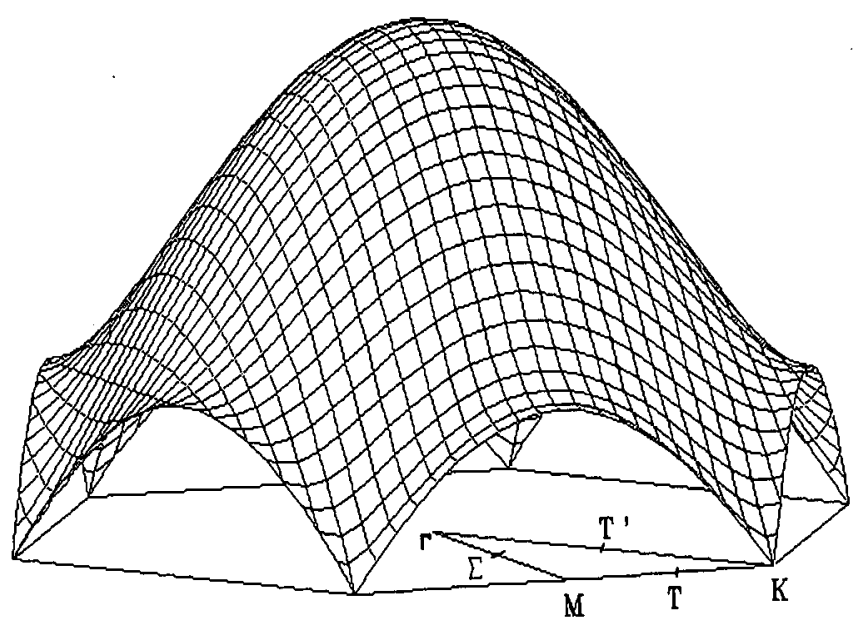

Fig. 2. The surface of the function $\left|\Gamma_{1}\right|$ within the Brillouin zone, for SC $\{111\}$ structure.

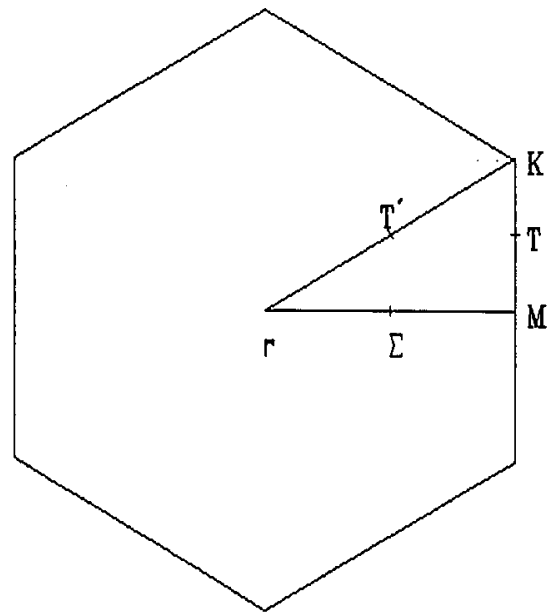

Fig. 3. A selected path of variations of the in-plane wave vector $k_{\|}$on the 1st Brillouin zone for SC $\{111\}$ structure with the characteristic points of the zone indicated thereon.

It turns out moreover that the physical factor is of essential importance which modifies the structure of the energy spectrum thus leading to hybridization. In Refs. [4] and [5] the role of such a factor was played by $a, c$ and $N$ : a variation in one of them separately led to the phenomenon under discussion. In this paper, we shall consider the possibility of hybridization varying $a$ and $c$ (at $N$ maintained constant) simultaneously; albeit $a$ and $c$ will not vary independently but rather 


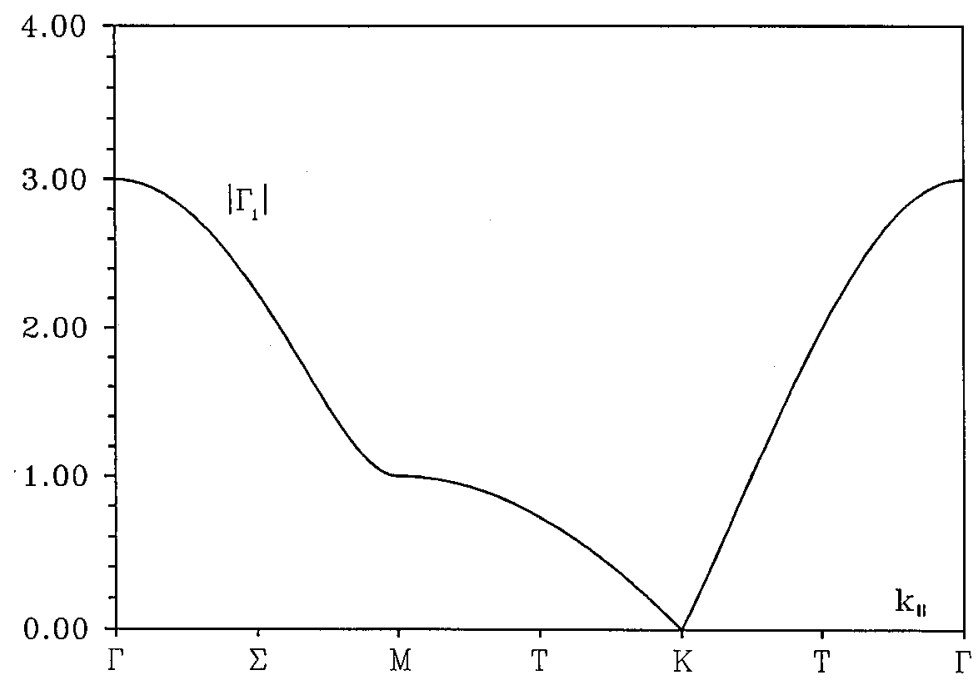

Fig. 4. Graph of the function $\left|\Gamma_{l}\right|$ versus $k_{\|}$as the latter varies along the path shown in Fig. 3.

both, interrelated by way of variations in $\left|\Gamma_{1}\right|$ (both $a$ and $c$ are functions of $\boldsymbol{k}_{\|}$, but it should be kept in mind that their ratio remains constant). For hybridization to occur the parameters $A_{1}$ and $A_{3}$ have to be chosen so that the $(c, a)$-trajectory shall intersect the so-called hybridization line in the $c-a$ diagram (see Puszkarski [5]). The phenomenon is not readily visible in the energy plot without having recourse to magnification. Accordingly, in Fig. 5, we have appropriately magnified the variations in energy at the places corresponding to hybridization.

In Fig. 5 we observe hybridization between symmetric states, whereas Figs. 6 and 7 enable us to follow the evolution of their profiles in the process of hybridization (profiles are taken for characteristic points of the zone and from the regions of hybridization - the "path" of the vector $k_{\|}$on the zone is shown in Fig. 3).

With varying $k_{\|}$, the localized states first "emerge" from the band and then hybridize, as it is clearly apparent from the "exchange" in localization nature of the profiles. The next essential point of the zone is $K$, where $\left|\Gamma_{1}\right|$ vanishes (causing the energy of the symmetric and antisymmetric states localized on the surface to become equal, as in Ref. [1]). Next, $\left|\Gamma_{1}\right|$ becomes greater than zero and hybridization takes place once again; we return to the starting point $\Gamma$, since the path on the zone is a closed path.

Figure 8 shows a $c-a$ diagram with the trajectory corresponding to the path shown in Figs. 3 and 4 and with the lines of hybridization. Moreover, the hyperbolae are plotted marking the regions of "generation" of the localized states thus providing a fuller interpretation of our results.

Figure 9 shows the regions of occurrence of localized states on the Brillouin 

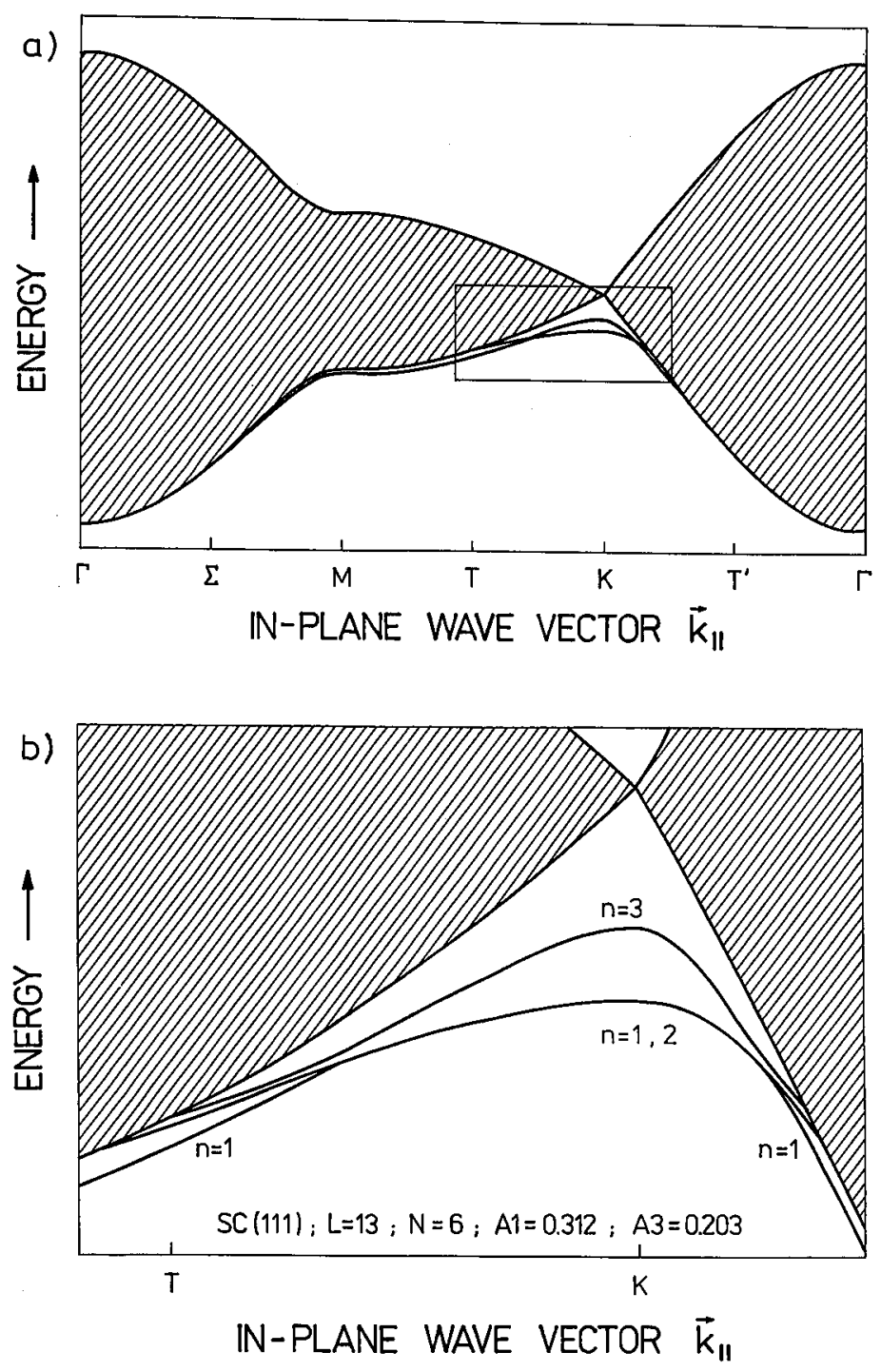

Fig. 5. (a) Changes in the energy of localized states versus the in-plane wave vector $\boldsymbol{k}_{\|\|}$(varying along the path of Fig. 3). (b) In the magnified inset we show the fragment of the graph proving the fact of hybridization under variations of $k_{\|}$. The level situated between the hybridizing levels corresponds to the antisymmetric state localized on the surface. The notations are those of Fig. 3. Values assumed are: $L=13, N=6, \dot{A}_{1}=$ $0.312, A_{3}=0.203$.

zone. Note that the number of localized states is the greatest in the regions where the function $\left|\Gamma_{1}\right|$ is close to zero. This is a universal property; it holds for all kinds of structures not discussed here, since it arises from the assumptions concerning the 

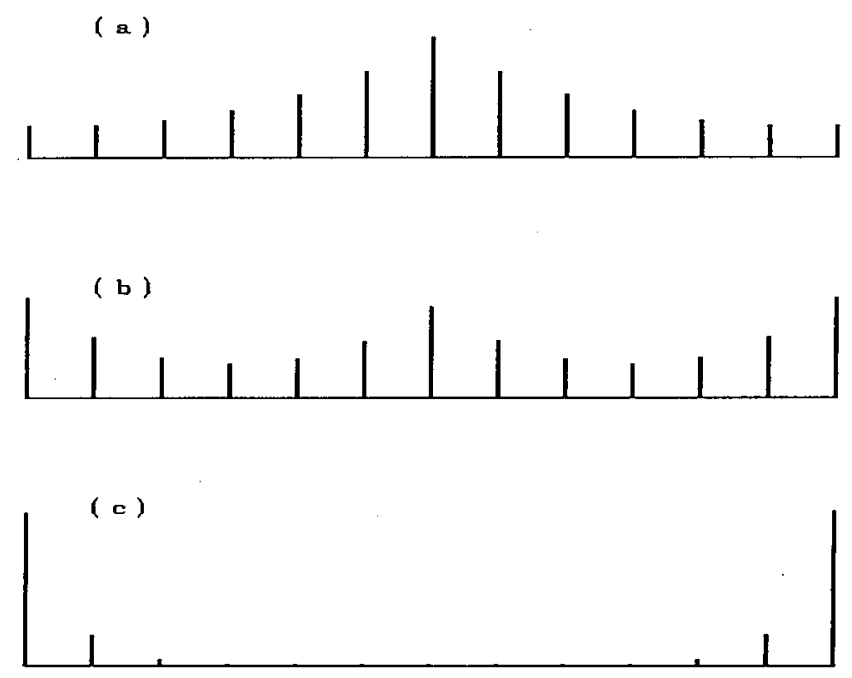

(d)
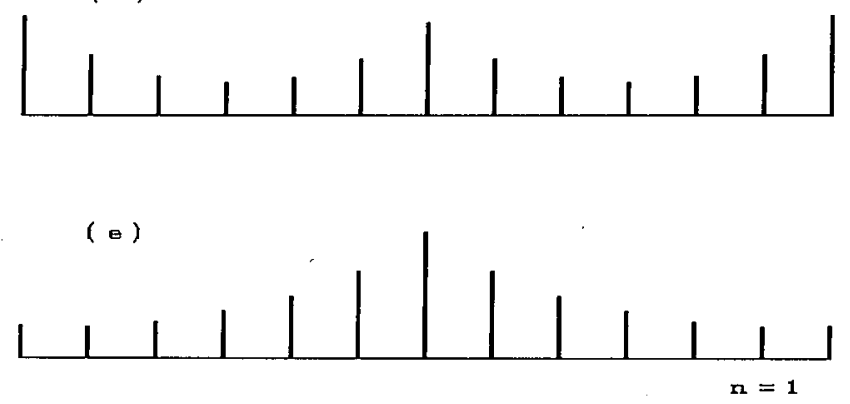

Fig. 6. The hybridization effect of mutual exchange of localization between the mode profiles ( $n=1$ and $n=3$ ). Profiles of the mode $n=1$ are shown in particular points along the variability path of $k_{\|}$plotted in Fig. 5:

(a) at the point of "emergence" from the band,

(b) at the point of first hybridization,

(c) at the point where $k_{\|}=K$,

(d) at the point of second hybridization,

(e) at the point of "hiding" into the band.

model and not from the crystallographical structure specified. The hybridization between localized states can occur if the direction of propagation of the waves in the plane of the film is chosen appropriately. When determining the vector $k_{\|}$for which the phenomenon shall occur, it should be noted that hybridization occurs only for parameters which ensure that the trajectory of variations on the $c-a$ diagram shall intersect the hybridization line (this is the necessary and sufficient condition). Hence the parameters should be relatively small in modulus (in order 

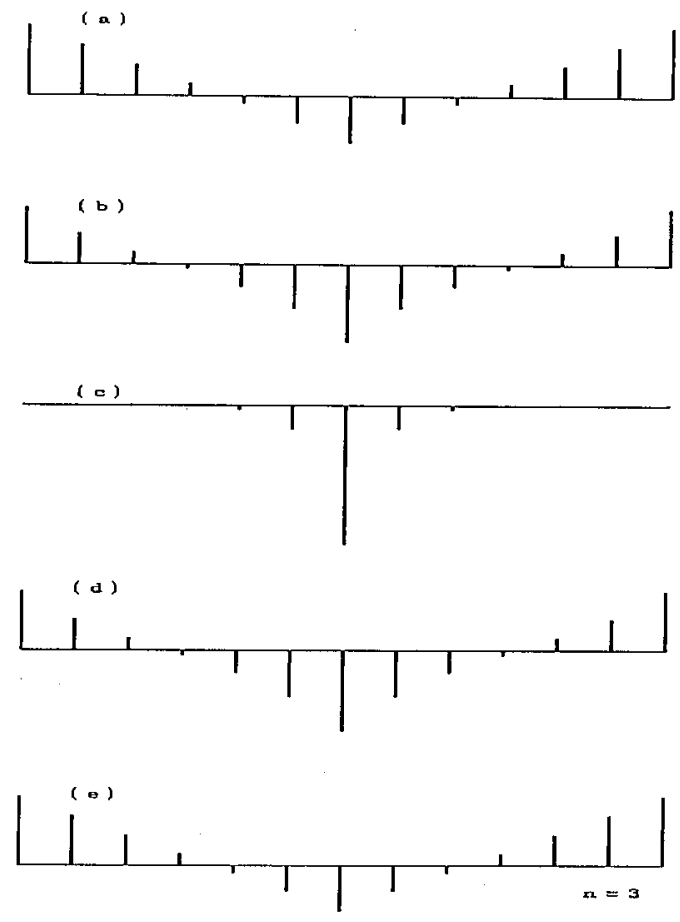

Fig. 7. Profiles of the mode $n=3$ for points identical with those of Fig. 6 .

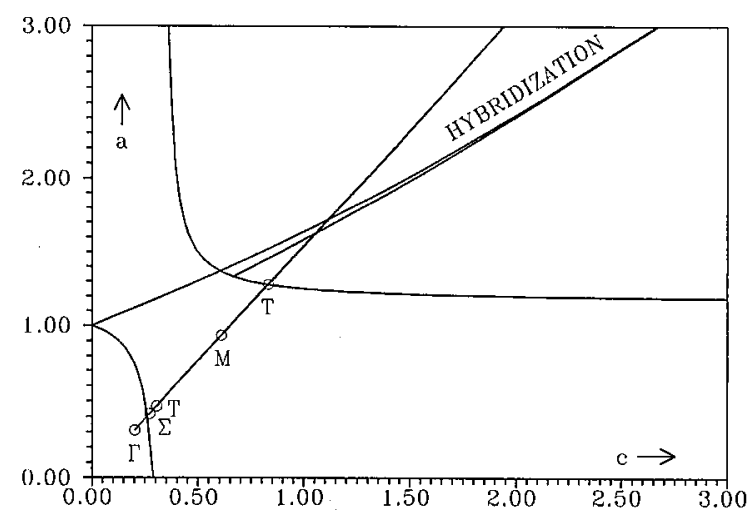

Fig. 8. $\quad c-a$ graph supplementing the information conveyed by Fig. 5. The hyperbola shown is the same as the one depicted in Fig. 1. The two lines marked as "hybridization" are composed of all those points on the $c-a$ graph which fulfill the necessary condition for hybridization. The places where localized states arise and hybridize are easily found by searching for the points where the trajectory intersects the respective lines. 


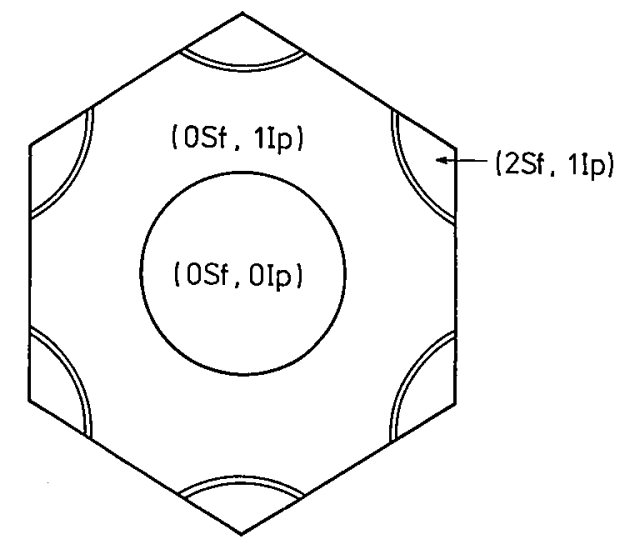

Fig. 9. Two-dimensional Brillouin zone with regions of existence of localized states indicated. The notation ( $m \mathrm{Sf}, n \mathrm{Ip}$ ) significs that in a given region $m$ states are localized on the surface and $n$ on the impurities. Note that the greatest number of localized states occurs in the vicinity of the zeros of $\left|\Gamma_{1}\right|$ and that the boundaries of the respective regions of existence of localized states result from the shape of the surface in Fig. 2. The parameter values used in plotting Fig. 9 are those of Fig. 5.

that the initial point of the trajectory shall lie below the hybridization line) whereas their ratio $A_{1} / A_{3}$ should be rather great (in order that the trajectory shall be steeper than the hybridization line).

\section{Conclusions}

We have dealt with an admixtured thin magnetic film, described by a classical Heisenberg IIamiltonian. The problem, owing to the symmetry imposed on the system, proved reducible to that of a one-dimensional system (a chain). Particularly, our interest bore on the occurrence of hybridization in a model of this kind under the effect of the variation of some dynamical factor, in contradistinction to the case of Refs. [4] and [5] where it occurred under the effect of parameters which, in an experiment, were generally constant quantities for a given specimen. We have found that the phenomenon searched for does in fact occur, albeit for certain specified orientations of the in-plane vector $k_{\|}$only, so that it should not be expected to be observable in experiment indifferently. Irrespective of the physical interpretation of the preceding considerations, it should be stated that the mathematics used in this work is applicable as well to other problems, e.g. to situations involving phonons, electrons, holes, etc. This enables us to predict that hybridization can take place (beside magnetic systems) in studies of electronic, phononic, and other states. 


\section{References}

[1] H. Puszkarski, Acta Phys. Pol. A38, 217 (1970).

[2] H. Puszkarski, Acta Phys. Pol. A71, 937 (1987).

[3] R.J. Jelitto, Z. Nat.forsch. A 19, 1567 (1964).

[4] H. Puszkarski, Prog. Surf. Sci. 25, 155 (1987).

[5] H. Puszkarski, Acta Phys. Superficierum 1, 75 (1990). 